\title{
Norois
}

Environnement, aménagement, société

$211 \mid 2009 / 2$

Eau, pêche, tourisme rural, conflits d'usage

\section{Eaux sous « pressions » et développement des territoires périurbains en Méditerranée occidentale (Languedoc-Roussillon) La vigne, le Sphaeromide raymondi et les piscines}

Water under "pressure" and developing outer-urban territories in the mediterranean Region. Vines, Sphaeromide raymondi and swimming pools

\section{Stéphane Ghiotti et Anne Rivière-Honegger}

\section{(2penEdition}

Journals

Édition électronique

URL : https://journals.openedition.org/norois/2866

DOI : $10.4000 /$ norois. 2866

ISBN : 978-2-7535-1559-8

ISSN : 1760-8546

Éditeur

Presses universitaires de Rennes

Édition imprimée

Date de publication : 1 octobre 2009

Pagination : $37-52$

ISBN : 978-2-7535-0937-5

ISSN : 0029-182X

Référence électronique

Stéphane Ghiotti et Anne Rivière-Honegger, «Eaux sous « pressions » et développement des territoires périurbains en Méditerranée occidentale (Languedoc-Roussillon) La vigne, le Sphaeromide raymondi et les piscines », Norois [En ligne], 211 | 2009/2, mis en ligne le 01 octobre 2011, consulté le 13 janvier 2022. URL : http://journals.openedition.org/norois/2866 ; DOI : https://doi.org/10.4000/norois.2866 


\title{
EAUX SOUS « PRESSIONS » ET DÉVELOPPEMENT DES TERRITOIRES périurbains en Méditerranée occidentale (LANGuedoc- Roussillon) La vigne, le SPHAERomide RAymondi et LeS PISCines
}

\author{
StéPHANe GHiotTI \\ FRE 3027 Mutations des Territoires en Europe \\ (Université Montpellier 3), \\ Bât BRED, Route de Mende - 34190 MontPELlier cedex 5, France \\ Stephane.ghiotti@univ-montp3.fr \\ Anne Rivière-Honegger \\ UMR 5600 CNRS Environnement Ville Société \\ (Université de Lyon), \\ 18 rue Chevreul - 69362 Lyon cedex 07, France \\ honegger@club-internet.fr
}

\section{RÉSUMÉ}

Cet article propose d'analyser les facteurs d'évolution des dynamiques territoriales relatives à l'eau et au développement en contexte méditerranéen où la rareté de l'eau se conjugue aujourd'hui avec de fortes mutations démographiques, économiques et environnementales. L'exemple de la moyenne vallée de l'Hérault est illustratif d'une situation de changement où se posent les questions d'eaux sous " pressions » et de développement; et permet d'identifier les germes des évolutions futures des modes de gestion.

MotS CLÉ : eau - politiques de l'eau - analyse comparative - approche diachronique - enquêtes - Méditerranée occidentale - région Languedoc-Roussillon-vallée de l'Hérault - périurbain

\section{ABSTRACT}

Water under "pressure" and developing outer-urban territories in the mediterranean Region. Vines, Sphaeromide raymondi and swimming pools

This article suggests analyzing the factors of the evolutions of the territorial dynamics relative to the water and to the development in Mediterranean context where the rarity of the water conjugates today with strong demographic, economic and environmental transformation. The changing situation in the middle valley of Hérault, where issues of water under pressure and development are raised, is an interesting case study; and allows to identify the germs of the future evolutions of the modes of management.

Key words : water - waterpolicy - comparative analysis - diachronic approach - interviews - western Mediterranean - region Languedoc-Roussillon - Hérault valley - outerurban territories 
La problématique développée est issue du croisement de deux réflexions menées en parallèle dans le cadre, pour la première d'entre elle, d'une expertise collective réalisée par l'INRA sur l'adaptation de l'agriculture à la sécheresse (INRA, 2006), pour l'autre d'une recherche sur la perception du risque environnemental en situation de crise hydraulique dans l'utilisation de l'eau agricole en Méditerranée occidentale (Puech et al., 2006) ${ }^{1}$. Ces recherches posent le postulat que les pressions, comme le risque, ne sont pas des phénomènes exogènes aux sociétés et que les solutions « pertinentes » ne se limitent pas à l'adaptation. Pour l'une comme pour l'autre, l'analyse du territoire de la moyenne vallée de l'Hérault est privilégiée.

Ce territoire cumule des pressions multiples sur la ressource en eau [démographique (besoins en eau potable et assainissement), économique (vente d'eau brute), environnementale (prospection et pompages dans les karsts), agricole (irrigation de la vigne), urbaine et périurbaine] et un paysage politico-institutionnel illustratif de 150 ans de gestion de l'eau en France confronté aux logiques d'aménagement et de développement des territoires. L'analyse des modalités d'articulation des logiques sectorielle et spatiale, des rapports de pouvoir local/local et local/central et des moyens financiers mobilisés constitueront des éléments d'interprétation concernant la question : quelles stratégies pour quelles eaux pour le Languedoc-Roussillon en 2015?

Plusieurs niveaux d'analyse ont été retenus pour saisir les enjeux et les jeux d'acteurs territorialisés liés à l'eau : celui de l'exploitation agricole, celui des dispositifs d'action au niveau local, celui du contexte régional et, au-delà, celui de la mise en application de la Loi directive Cadre européenne sur l'eau (2000) 2 ${ }^{2}$. La démarche est qualitative et repose sur l'utilisation de deux guides différenciés d'entretien, l'un auprès d'une quinzaine d'agriculteurs et l'autre concernant l'ensemble des acteurs institutionnels ou associatifs. Les entretiens sont complétés par la lecture systématique des textes législatifs sur l'eau et des dossiers préalables, contractuels ou d'évaluation des contrats de rivière, des Schémas d'aménagement et de gestion des eaux (SAGE) et des opérations de développement territorial mises en place sur le territoire régional.

Dans un premier temps, nous nous interrogerons sur la nature, les formes et les rythmes d'évolution des dynamiques territoriales relatives à l'eau à un pas de temps séculaire, puis nous examinerons des problématiques particulières en lien avec les fortes mutations contemporaines du territoire - la viticulture, la préservation et le maintien des milieux aquatiques, l'augmentation des besoins en eau potable intra et extra muros - ce qui nous permettra d'apporter des éléments de réflexion sur les questions sous-jacentes de pressions sur les ressources, de crise hydraulique et de processus de construction d'une conscience commune de l'eau.

\section{Eaux sous pressions, territoires sous pressions : 150 ans d'évolution}

\section{UNE IRRÉGULARITÉ DES RESSOURCES DANS LE TEMPS ET DANS L’ESPACE}

L'eau est abondante dans la région : $400 \mathrm{~m}^{3}$ environ en sortent à chaque seconde et rejoignent la Méditerranée ou alimentent les fleuves du versant atlantique. En outre, grâce à une concession obtenue en 1956 par la Compagnie nationale d'aménagement de la région du Bas-Rhône Languedoc (CNABRL) pour une durée de 75 ans (décret du 14 septembre 1956), le canal du Bas-Rhône - ou canal Philippe LAMOUR - apporte à la région au maximum un débit de $75 \mathrm{~m}^{3} / \mathrm{s}$ (BRL, 2008). Mais cette richesse n'est pas aisément mobilisable, en raison notamment des pentes fortes et de l'irrégularité hydroclimatique annuelle et interannuelle. Des totaux annuels d'apparence normale (700 à $800 \mathrm{~mm}$ ) cachent en fait des pluies violentes et concentrées sur quelques jours, qui souvent dégradent plus les sols qu'elles ne favorisent la végétation. Cette première

1. CNRS-SHS, Programme «Eau, environnement, sociétés », Crises hydrauliques et perceptions du risque environnemental en Méditerranée occidentale (2003-2006). Ce programme, coordonné par le laboratoire Ladyss du CNRS, a associé quatre pays méditerranéens : Espagne, France, Maroc et Tunisie.

2. Directive Cadre Européenne sur l'eau 2000/60/CE du 23 octobre 2000, transposée en droit interne par la loi n 2004-338 du 21 avril 2004. 
caractéristique a pour conséquence de créer des tensions récurrentes et croissantes entre besoins et disponibilités de la ressource. À cela, il faut ajouter la variabilité spatiale de l'apport pluvial : «Abondante en montagne, absente en garrigue, surabondante dans la plaine » souligne R. Ferras (1998). Ce qui se traduit respectivement en termes de gestion par « une abondance bénéfique, une absence gênante et un excès maléfique ».

Le régime hydrologique de type méditerranéen «trop d'eau, pas assez d'eau » caractérise la plupart des cours d'eau de la région. L'importante variation des débits est marquée par des crues d'automne et de printemps brusques et abondantes, un étiage estival très marqué avec des risques de crues liés aux précipitations orageuses, lesquelles sont qualifiées par M. Pardé (1925) « d'énormes, foudroyantes, dévastatrices ». Il en résulte une ressource non fiable en période estivale. Presque tous les cours d'eau de la région ont, de juin à septembre, des débits inférieurs au dixième du module annuel qui est le référent pour la loi Pêche (1984) - et en cela sont assimilables à de véritables oueds. Seul le Rhône constitue une ressource régulière. Cela explique que d'importants ouvrages de transfert et de stockage de la ressource aient été développés au cours des dernières décennies. Globalement, la qualité des eaux superficielles est moyenne voire bonne en LanguedocRoussillon (Agence de l'eau Rhône-Méditerranée et Corse, 2005). Elle est en voie d'amélioration depuis quinze ans malgré des points noirs persistants. Ces derniers sont situés à l'aval des cours d'eau au sein des espaces fortement anthropisés du littoral et au contact des principales nappes aquifères de la région. Ainsi, les eaux souterraines sont soumises à de fortes pressions quantitatives (nappe de l'Astien) ${ }^{3}$ et qualitatives (nappe de l'étang de l'Or) dans cette partie du territoire où se conjuguent urbanisation, activités touristiques et infrastructures.

\section{UN TERRITOIRE RÉGIONAL EN MUTATION}

Les projections les plus récentes estiment, en effet, l'augmentation de la population en Languedoc-Roussillon à 800000 personnes à l'horizon 2030 (INSEE, 2005). Les secteurs les plus concernés seront la frange littorale mais également les zones de garrigues de l'arrière-pays où la multiplication des fonctions et des usages accroît considérablement la pression sur les ressources, notamment l'eau et le foncier. Le territoire du bassin-versant de l'Hérault connaît ainsi un accroissement démographique soutenu (augmentation de la population de $25 \%$ entre 1982 et 1999, par exemple, selon les données du Recensement général de la population).

À l'échelle du Languedoc-Roussillon, l'agriculture demeure un secteur clé de l'activité régionale avec $6 \%$ de l'emploi marchand sur 44000 exploitations (Pôle prospective, 2007). Mais les terres agricoles sont soumises à de fortes pressions et leur avenir repose sur plusieurs incertitudes telles que la mondialisation des marchés, la transformation des structures de production et l'évolution de la Politique agricole commune (PAC) même si la vigne est peu concernée. La superficie agricole totale a reculé d'1 \% par an entre les deux derniers recensements agricoles (1988-2000). La crise agricole a favorisé la conversion de la vigne notamment en lotissements. L'économie dépend ainsi de façon croissante de l'activité résidentielle et des services générés par la croissance démographique. L'extension du tissu urbain repose principalement sur le prix du foncier, la qualité des espaces environnants et la desserte. Chaque année la construction des maisons individuelles consomme en moyenne près de 1000 hectares dans la plaine agricole languedocienne (Pôle prospective, 2007). Logique agricole et logique urbaine s'expriment à travers des conflits d'usage. Parmi les autres facteurs endogènes et exogènes qui pourraient entraîner des situations de blocages figure le niveau des ressources en eau (Genin, 2005).

Ces dynamiques démographiques et territoriales marquées par la déprise agricole, le renforcement des infrastructures routières (autoroute A75 qui relie Paris à la Méditerranée) et par une recomposition entre les espaces ruraux et urbains prennent et prendront place dans un environnement institutionnel (décentralisation, compétitivité des territoires) et législatif (Politique Agricole

3. La nappe de l'Astien est gérée par le Syndicat mixte d'études et de travaux de l'Astien : SMETA. 
Stéphane Ghiotti, Anne RiviÈre-Honegger

Commune, Loi sur l'eau et les milieux aquatiques ${ }^{4}$, Directive cadre européenne) qui, nous en faisons l'hypothèse, augmentera les contraintes pour l'accès et le partage des ressources. À titre d'exemple, si l'atteinte du bon état écologique pour les masses d'eau à l'horizon 2015 privilégie une approche par la qualité, les caractéristiques climatiques des territoires méditerranéens font que l'atteinte des objectifs annoncés passera par la régulation des déséquilibres quantitatifs. L'heure n'est plus à la satisfaction des différents usages mais au partage d'un volume d'eau au sein d'un bassin, ce qui change très sensiblement la problématique, d'autant plus fortement que les priorités vont à l'alimentation en eau potable et à l'environnement (débit d'étiage).

\section{DES TERRITOIRES HYDRAULIQUES MULTIPLES}

La gestion du déficit hydrique a fait l'objet, au cours des siècles, d'aménagements hydrauliques remarquables visant à transférer l'eau, d'abord au sein d'un même bassin-versant, avec les systèmes de canaux d'arrosage que l'on trouve en particulier dans les Pyrénées-Orientales, puis à l'échelle de plusieurs de ces bassins. Retracer la genèse des politiques de l'eau permet d'identifier, par la suite, trois temps : celui du passage de la gestion individuelle à la gestion collective, celui de la mise en place des sociétés d'aménagement et celui de la gestion concertée par bassin-versant. Aujourd'hui, le constat est celui d'une multiplication des acteurs et des démarches qui suscite une réflexion sur les modalités d'une nouvelle gouvernance. La vision départementale et régionale pour l'eau est contenue dans le document prospectif Aqua 2020 (BRL, 2006).

\section{D’une gestion individuelle à une gestion collective : les Associations syndicales autorisées}

La gestion de l'eau, comme facteur vital de production, et non d'intensification, est un enjeu important. À l'échelle de la région, les surfaces équipées pour l'irrigation représentent $15 \%$ de la surface agricole utilisée. L'une des caractéristiques est la sous-utilisation des équipements existants, ce qui peut être rattaché à une marge de sécurité nécessaire dans un contexte climatique méditerranéen. Les réseaux collectifs sont majoritaires (RGA, 1999) : 30 \% seulement des exploitations irriguent à partir de ressources individuelles, contre $60 \%$ à l'échelle nationale. « La territorialisation de la gestion de l'eau apparaît comme une forme d'organisation sociale ancienne et complexe, en lien direct avec les luttes politiques, institutionnelles et scientifiques pour l'appropriation de la ressource et de sa gestion » (Ghiotti, 2007). En Languedoc-Roussillon, cela prend la forme d'associations de propriétaires fonciers : les Associations syndicales autorisées ${ }^{5}$. Plus de 700 sont encore en activité, comme le montre leur inventaire (Puech et Rivière-Honegger, 2001 ; Rivière-Honegger, 2004). On peut considérer qu'environ 36000 hectares irrigables gérés par un peu plus de 300 ASA sont en irrigation gravitaire, soit environ $22 \%$ de la superficie irrigable de la région (Rivière-Honegger, 2001). L'association de Gignac, la plus importante (en nombre d'adhérents) du département de l'Hérault et l'une des plus anciennes est, comme nous allons le voir, symptomatique de l'évolution de la gestion de l'eau dans le Midi de la France.

\section{La mise en place des sociétés d'aménagement : Bas-Rhône Languedoc ( $6 \mathrm{~mm}^{3} / a n$ prélevés)}

La décennie 1950-1960 marque un véritable tournant puisqu'elle inaugure le développement et l'aménagement volontariste du territoire sur des bases régionales. Le décret du 30 juin 1955 consacre la régionalisation du Plan ${ }^{6}$. Il est fondamental en ce qu'il institutionnalise l'échelle régionale comme une échelle d'action et qu'il fait de l'eau et de l'urbanisme les pièces maîtresses de sa politique par la création des Sociétés d'aménagement régionales. La Compagnie nationale

4. Loi n 2006-1772 sur l'eau et les milieux aquatiques du 30 décembre 2006, JORF du 31 décembre 2006.

5. Les ASA, dont l'origine remonte à la loi du 21 juin 1865, sont des établissements publics réunissant des propriétaires privés. Elles sont traditionnellement dédiées à l'irrigation mais aussi au drainage des terres et à la gestion des rivières. Elles sont caractérisées par une grande diversité de taille, un fonctionnement largement tributaire du bénévolat et une implication croissante des collectivités locales dans leur gestion.

6. Décret 55-876 du 30 juin 1955 relatif aux sociétés de développement régional (JO du 2 juillet 1955). 
d'aménagement de la région du Bas-Rhône Languedoc (CNABRL) est ainsi créée en 1956. Le territoire à équiper a été divisé en deux zones, la zone Est, tributaire du Rhône et la zone Ouest, dépendante des fleuves côtiers (Hérault, Orb et Aude) et dont l'alimentation en eau repose sur la réalisation de barrages.

Dans les années 1990, la superficie équipée de la concession d'État approche 100000 hectares. Celle relevant des concessions départementales et particulières est de 54400 hectares. La superficie irriguée représente, depuis la fin des années 1960, environ $30 \%$ de la superficie régionale irriguée. Environ 30000 hectares sont irrigués (Carrière, 1999). Le partage équitable de la ressource entre tous les usages, enjeu majeur pour le développement de la région, connaît aujourd'hui une acuité particulière dans un contexte d'élargissement des activités, des acteurs et des territoires. Ainsi, Bas-Rhône Languedoc affirme aujourd'hui son rôle d'acteur majeur dans la production d'eau brute ${ }^{7}$ à destination d'usages urbains et touristiques. En se détournant de sa mission agricole première, la Compagnie s'engage dans la même démarche que la Société du canal de Provence, trente ans plus tôt, en Région Provence-Alpes-Côte-d'Azur. Cette évolution se fait en partenariat avec les collectivités territoriales, régionales notamment, dont les compétences dans ce domaine ont été élargies suite à la loi de décentralisation du 13 août $2004^{8}$.

\section{Des démarches et des projets territoriaux à articuler}

\section{- Vers une gestion concertée : du Contrat de rivière au SAGE}

Le bassin-versant du fleuve Hérault est un exemple intéressant de cette évolution. Il s'étale sur $2500 \mathrm{~km}^{2}$, entre deux départements, le Gard et l'Hérault, à travers 166 communes, et concerne 150000 habitants. C'est un territoire contrasté car il se situe entre les Cévennes, les Causses, la plaine. Il recèle deux ressources principales inégalement réparties. Les nappes alluviales de l'Hérault, très sollicitées, fournissent $75 \%$ de la production en eau potable du bassin ${ }^{9}$ et les karsts moins exploités mais de plus en plus convoités (CG34, 2005). Des barrages réservoirs à l'influence locale ont été construits.

Après un contrat de rivière, le Schéma d'aménagement et de gestion de l'eau du fleuve ${ }^{10}$ Hérault a été initié par les départements du Gard et de l'Hérault avec l'État et les acteurs locaux face au constat d'acteurs nombreux et d'actions parcellisées et peu coordonnées. Un périmètre a été délimité en 1999.

La gestion quantitative est l'enjeu majeur de la demande de gestion locale de l'eau pour le SAGE Hérault ${ }^{11}$. Le principal constat est « qu'il n'y a pas de globalité de la gestion, ni au sens territorial, puisque le territoire est découpé en une multitude d'entités, ni au sens des domaines d'activités,

7. L'eau dite «brute » est une eau non traitée, destinée à l'arrosage des jardins et au remplissage des piscines.

8. Par exemple, la région Languedoc-Roussillon a récupéré par une convention qui fixe les modalités du transfert, le réseau hydraulique concédé par l’État à la Compagnie BRL.

9. Le prélèvement total sur le bassin s'élève à 64 millions de $\mathrm{m}^{3} / \mathrm{an}$, dont $56 \%$ pour l'eau potable et $44 \%$ pour l'irrigation.

10. Les articles 3 et 5 de la loi sur l'eau de 1992 définissent les nouveaux schémas d'aménagement et de gestion des eaux : Le Schéma directeur d'aménagement et de gestion des eaux (SDAGE) est élaboré dans le cadre des grands bassins hydrographiques tels que le Bassin Rhône-Méditerranée-Corse, par exemple, et le Schéma d'aménagement et de gestion des eaux (SAGE) est proposé à l'échelle des bassins versants des cours d'eau.

SDAGE : Schéma directeur d'aménagement et de gestion des eaux (article 3) «Un ou des schémas directeurs d'aménagement et de gestion des eaux fixent pour chaque bassin ou groupement de bassin les orientations fondamentales d'une gestion équilibrée de la ressource en eau, telle que prévue à l'article $1^{\text {er. }}$ »

SAGE : Schéma d'aménagement et de gestion des eaux (article 5) «Dans un groupement de sous-bassins ou un sous-bassin correspondant à une unité hydrographique ou à un système aquifère, un schéma d'aménagement et de gestion des eaux fixe les objectifs généraux d'utilisation, de mise en valeur et de protection quantitative et qualitative des ressources en eau superficielle et souterraine et des écosystèmes aquatiques ainsi que de préservation des zones humides, de manière à satisfaire aux principes énumérés à l'article $l^{\text {er }}$.

CLE : la Commission locale de l'eau est créée au titre de la Loi sur l'eau de 1992. Lélaboration du SAGE exige la création d'une CLE, véritable parlement de l'eau qui implique tous les acteurs concernés dans l'élaboration de programmes et les processus de décision.

11. Une mission d'animation a été créée en septembre 2002 et la Commission locale de l'eau installée en janvier 2003. Celleci n'a pas d'existence juridique et n'assure donc pas de maîtrise d'ouvrage. Les travaux de la CLE, qui travaille dans le cadre d'une concertation ouverte avec tous les acteurs de l'eau du bassin, ont abouti à la rédaction et à la validation d'un 
puisque la gestion demeure essentiellement sectorielle [...]. Il n'y a donc pas de politique globale de gestion du fleuve Hérault, pas de vision d'ensemble, pas non plus d'anticipation des problèmes à l'échelle pertinente du bassin. » (CG34, 2005, p. 6).

Les orientations dégagées par la CLE à l'issue de l'élaboration du diagnostic sont au nombre de quatre: «Mettre en œuvre une gestion quantitative durable permettant de satisfaire les usages et les milieux; maintenir ou restaurer la qualité de la ressource et des milieux pour permettre l'expression de leur potentialité biologique et leur compatibilité avec les usages; limiter et mieux gérer le risque inondation et développer l'action concertée et améliorer l'information. » (CG34, 2005).

Tout SAGE adopté a une portée réglementaire ${ }^{12}$. Le SAGE Hérault concrétise une nouvelle façon de gérer l'eau ${ }^{13}$. Face aux pressions multiples et croissantes sur les ressources, l'orientation est de définir des débits d'étiages de référence en différents points du bassin-versant afin de concilier les usages et d'assurer le bon fonctionnement des milieux aquatiques.

- La multiplication des ailleurs : quelle dialectique entre territoire de gestion et territoires de décision?

En matière de gouvernance territoriale, les lois Chevènement ${ }^{14}$ et Voynet ${ }^{15}$ ont initié de nouveaux périmètres de gestion des coopérations intercommunales. Les schémas de cohérence territoriale (SCOT), qui font une place importante à la préservation des espaces naturels et agricoles (Jarrige et al., 2006), sont des projets de planification concertée. De nouveaux outils réglementaires (Plan locaux d'Urbanisme, Plan de prévention du risque ${ }^{16}$, etc.) et la loi du 23 février 2005 relative au développement des territoires ruraux permettent à l'agriculture de devenir une composante des nouveaux territoires urbains.

En matière de gestion de l'eau, l'approche par bassin est renforcée par le mouvement de recomposition organisationnelle et politique en cours, mais elle doit aussi s'articuler aux autres territoires en construction ou en développement dont il convient de souligner la diversité, souvent la profondeur historique et la légitimité sociale. «À côté du territoire de l'eau (ré?) émergent ou se constituent des territoires de l'eau plus en phase avec les logiques intercommunales, départementales et régionales articulées autour de la décentralisation qu'en lien avec les approches naturelles ou environnementales » (Ghiotti, 2007).

Une action transversale existe ainsi entre les bassins versants de l'Orb et de l'Hérault à l'initiative de la structure de gestion de l'Orb ${ }^{17}$ sur le thème de la ressource en eau (fig. 1). C'est une démarche originale qui suscite l'intérêt des instances de Bassin. Mais, dans ce contexte particulier, les présidents de structures de gestion sont difficilement en mesure d'imposer leur point de vue face à l'aménageur BRL dont c'est un secteur de distribution d'eau. Ceci pose la question des lieux d'arbitrages. Si la coordination technique fonctionne, la définition des règles de péréquation financière reste entière. Il est à noter que la région Languedoc-Roussillon est relativement absente

état des lieux et d'un diagnostic, ainsi qu’à la définition des premières orientations du SAGE. L'ensemble a été validé par la CLE le 19 décembre 2005 et présenté au Comité de bassin le 20 janvier 2006.

12. Il est ainsi opposable au tiers et donc à l'administration et aux documents d'urbanisme (LEMA n 2006-1772 du 30 décembre 2006, article 77-2).

13. Le bassin-versant de l'Hérault est un bassin classé « prioritaire pour une amélioration de la gestion quantitative » dans le SDAGE RMC (1996). Dans le nouveau SDAGE (2009), il est divisé en 13 masses d'eau pour lesquelles sont assignés des objectifs à l'horizon 2015 dans le cadre de l'application de la Directive cadre européenne sur l'eau (2000).

14. La loi n 99-586 du 12 juillet 1999 relative au renforcement et à la simplification de la coopération intercommunale, dite Loi Chevènement.

15. La loi nº 99-533 du 25 juin 1999 d'orientation pour l'aménagement et le développement durable du territoire et portant modification de la loi n ${ }^{\circ}$ 95-115 du 4 février 1995 d'orientation pour l'aménagement et le développement du territoire, dite Loi Voynet.

16. Institué en 1987, modifié par la loi n 95-101 du 2 février 1995 relative au renforcement de la protection de l'environnement (Loi Barnier), le dispositif des PPR a été consolidé par la loi n² 2003-699 du 30 juillet 2003 relative à la prévention des risques technologiques et naturels (Loi Bachelot).

17. Syndicat mixte de la vallée de l'Orb : SMVO. 
de la gestion des structures (Syndicats mixtes notamment) ${ }^{18}$ contrairement à d'autres régions en France, ou à d'autres partenaires, comme le Conseil général, par exemple dans l'Hérault.

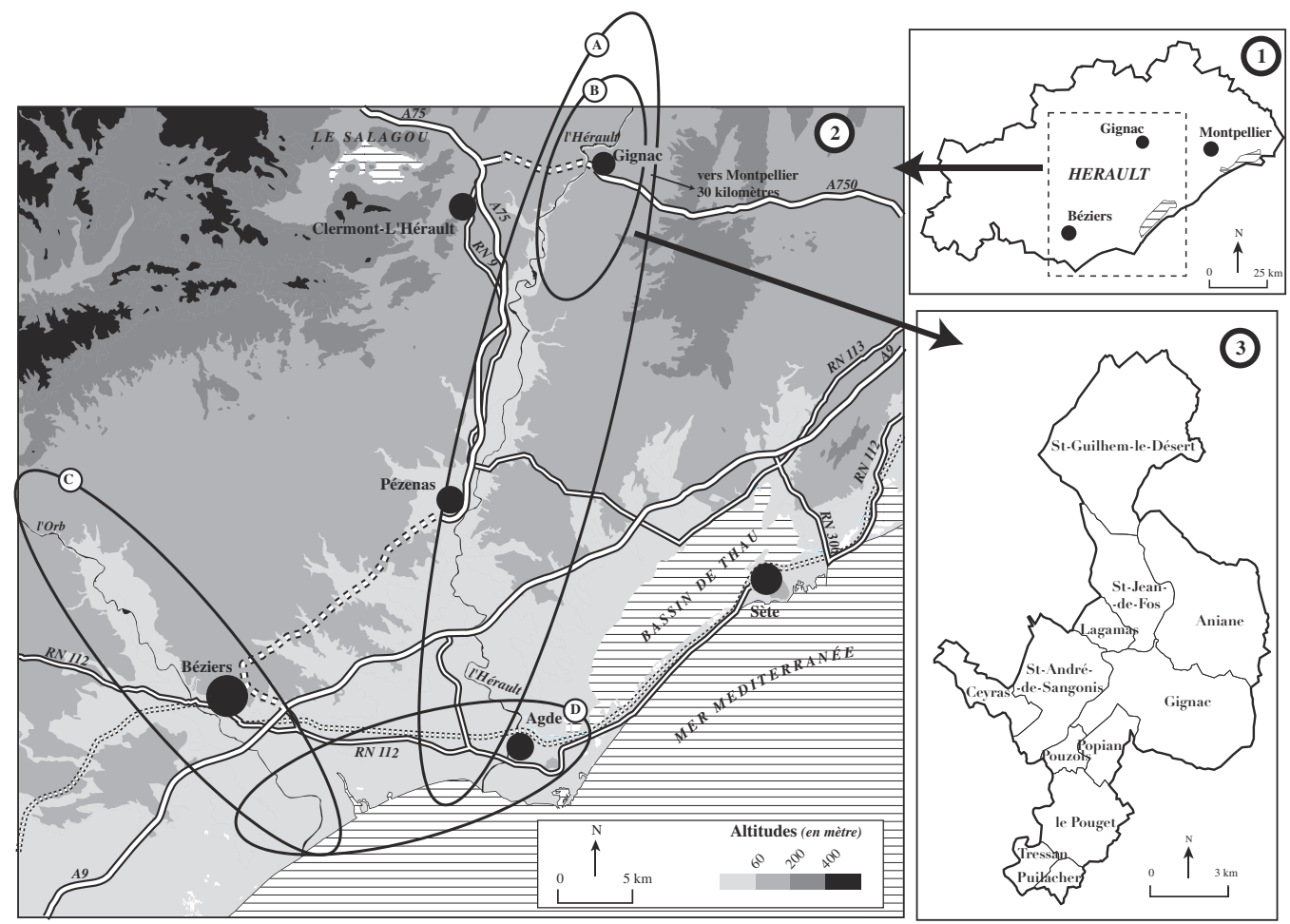

\begin{tabular}{|llll|}
\hline Gestion de l'Eau & & \multicolumn{1}{c|}{ Axes de Transports } \\
(A) Périmètre SAGE Hérault & & $=$ & Autoroute \\
(B) ASA de Gignac & & Route nationale \\
(C) Périmètre SMVO & & \\
(D) Périmètre SMETA & & & \\
\hline
\end{tabular}

Figure 1 : L'ASA du canal de Gignac et les syndicats du bassin-versant de l'ouest Hérault ASA of the channel of Gignac and Syndicates of the paying pond western the Herault

Dans le débat en cours, il convient ainsi d'être attentif à deux éléments. D'une part, il importe de se méfier de la surenchère sur les besoins futurs en eau. Les estimations émises par les différents utilisateurs ont doublé, voire triplé, par rapport aux premières estimations. D'autre part, il y a nécessité de rappeler qui a légitimité pour parler de l'eau, en l'occurrence les services de l'État et les gestionnaires de l'eau. Le territoire de la moyenne vallée de l’Hérault reflète bien cette complexité des processus et des solutions proposées pour y faire face.

18. En revanche, la région Languedoc-Roussillon n'est pas absente de la gestion de l'offre à travers la gestion des infrastructures BRL. 


\section{À l'échelle d'un périmètre : l'exemple de l'ASA de Gignac}

L’ASA de Gignac s'est érigée au fil des ans comme un véritable laboratoire de terrain dans notre processus de recherche. L'observation continue, depuis plus de dix ans, permet d'aborder des problématiques particulières en lien avec les fortes mutations de ce territoire et, au-delà, du territoire régional. Les tendances relevées et les questionnements ont souvent valeur générale.

\section{UNE GESTION COLLECTIVE ANCIENNE}

Situé à une trentaine de kilomètres au nord-ouest de Montpellier (fig. 1), cet aménagement centenaire est né de la crise du phylloxéra. Le canal d'irrigation de Gignac fait partie de l'histoire et de la vie de onze communes de la Moyenne Vallée de l'Hérault ${ }^{19}$ d'où il prend sa source (commune de Saint-Guilhem-le-Désert). Il permet, grâce à $56 \mathrm{~km}$ de voies à grand gabarit, $70 \mathrm{~km}$ de canaux secondaires et $250 \mathrm{~km}$ de rigoles, d'irriguer 3000 ha de vignes, de cultures et de jardins. Ces éléments font de l'ASA du canal de Gignac, chargée de sa gestion, l'une des plus importantes de la région. Le nombre d'utilisateurs s'élève à 3010 , répartis comme suit : 1800 adhérents agricoles, 1200 adhérents pour des jardins et 10 adhérents divers (coopératives, gravières, etc.) (ASA, 2005).

\section{UN ENJEU MAJEUR : GÉRER DE NOUVELLES FONCTIONS ET DE NOUVEAUX ACTEURS}

Aujourd'hui, dans un contexte de périurbanisation, de prise de conscience par la société des valeurs environnementales et de l'établissement d'une réglementation plus stricte dans ce domaine, le canal peut sembler en décalage avec les mutations de la vie économique et sociale de la région. Il reste en même temps un élément indispensable à la vie de la plupart des familles d'adhérents agricoles de l'association. Sans lui, bien des exploitations agricoles ne seraient pas économiquement viables.

Le $\mathrm{xx}^{\mathrm{e}}$ siècle est, dans l'histoire du canal, caractérisé par la croissance de la viticulture, la diversification agricole et le maintien en l'état de l'ouvrage existant sans remise en cause. Différents modes d'irrigation (gravitaire, basse pression et sous pression) offrent la possibilité d'une grande diversification des cultures. L'irrigation est à $93 \%$ gravitaire à la raie. Malgré les fortes évolutions des dernières décennies ${ }^{20}$, la viticulture reste la culture principale et, en matière de gestion de l'eau agricole, les viticulteurs demeurent les principaux interlocuteurs. $88 \%$ des usages de l'eau du canal servent encore à la production agricole (fig. 2). Les interrogations pour l'avenir sont cependant fortes, dans un contexte de surproduction chronique et de mévente.

Depuis deux décennies les associations de propriétaires fonciers connaissent un tournant dans leur histoire. L'irrigation n'est plus aujourd'hui la seule fonction assurée par les réseaux. Ces derniers permettent en particulier la fourniture d'eau brute pour des usages non agricoles. On assiste à un élargissement des fonctions assurées par le canal, des acteurs concernés et de l'échelle de gestion. Les conditions d'exploitation des réseaux s'en trouvent modifiées, ce qui est parfois la source de conflits et de concurrences. Par exemple, les usagers urbains aspirent à disposer d'eau avec un minimum de pression et sans être contraints par des tours d'eau ${ }^{21}$ La prise en charge financière des ASA (Puech, 2001 ; Garin et Loubier, 2002) est ainsi devenue de plus en plus difficile.

19. Tressan, Aniane, Gignac, Pouzols, Popian, Le Pouget, Puilacher, Ceyras, Saint-Jean-de-Fos, Saint-André-de-Sangonis, Lagamas.

20. Sur le périmètre de l'association la superficie occupée a reculé de 1739 hectares entre 1979 et 2000 (RGA, 2000). L'influence des primes d'arrachage est perceptible, renforcée par une forte demande de foncier pour la construction de lotissements. La diversification et l'apparition de nouvelles spécialisations maraîchères (melons par exemple) peuvent aussi être une source d'explication. Le nombre d'exploitations viticoles est en forte baisse : il est passé de 1270 en 1979 à 722 en 2000. (SCEES, recensement général agricole, 1979, 1988, 2000).

21. Les tours d'eau organisent la distribution. Les cahiers d'arrosage sont établis en période de chômage du canal, le tour d'eau se décalant d'un jour et 4 heures chaque année. Tout au long de la saison d'irrigation, 100 modules de 35 litres/seconde 


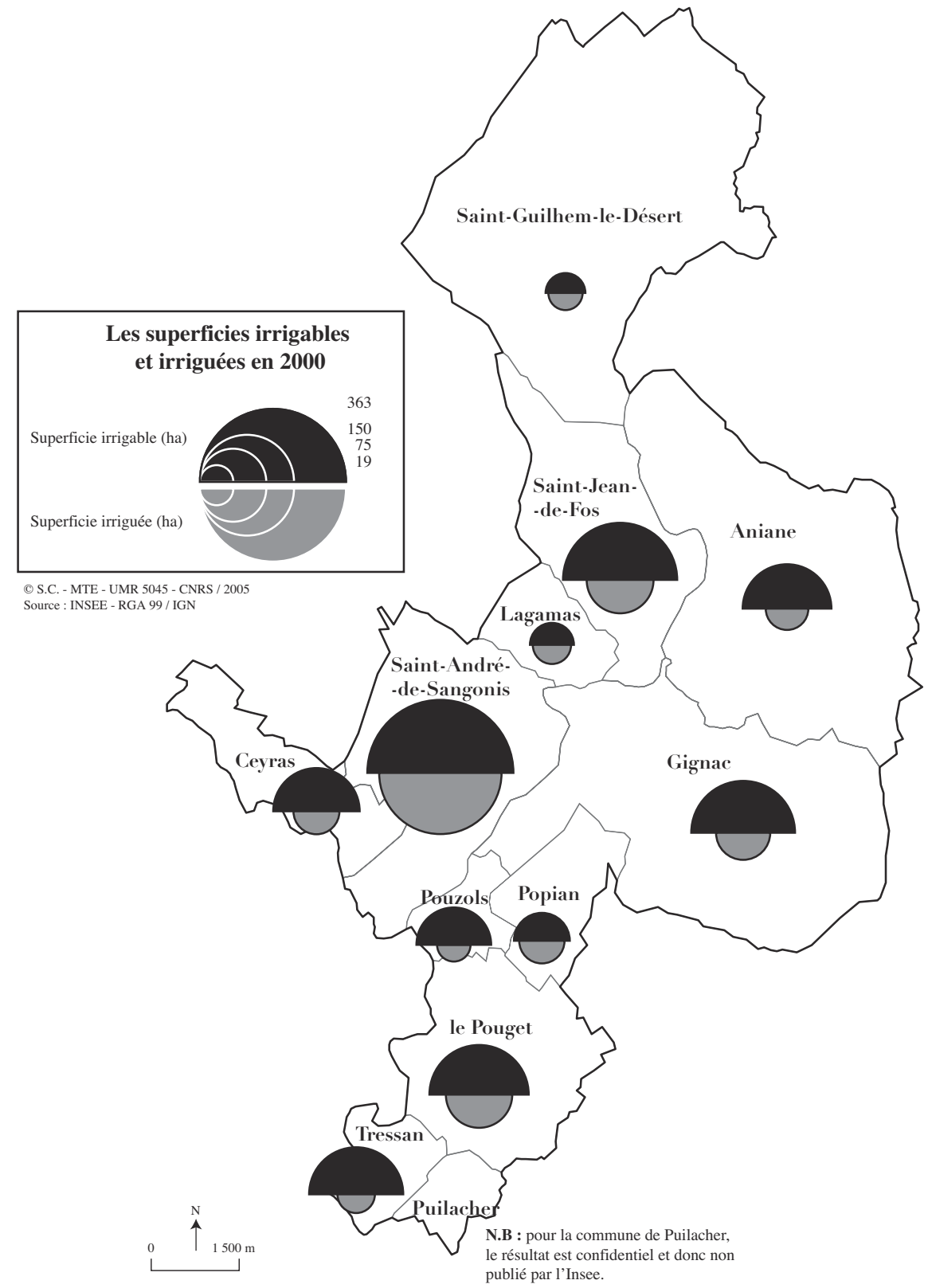

Figure 2 : L'irrigation au sein du canal de Gignac

The irrigation within the channel of Gignac

chacun sont successivement affectés aux terres irrigables sous contrôle des gardes canaux. La rotation se pratique en continu jour et nuit sur les 168 heures de la semaine selon le Règlement pour le service des arrosages et la police du canal approuvé en 1897. On observe aujourd'hui des aménagements. L'eau est ainsi coupée entre minuit et 5 h00 du matin. Depuis la campagne d'irrigation 2006 est expérimentée une alternance de la distribution d'eau non plus rive droite/rive gauche mais amont/aval sur un rythme 3 jours 3 jours. 
Stéphane Ghiotti, Anne RiviÈre-Honegger

\section{De NOUVEAUX USAGES, SOURCES DE PRESSIONS}

Alors que certains usages (industriels) sont aujourd'hui délaissés (usines hydroélectriques, gravières) car il n'est plus délivré de nouvelles autorisations, d'autres émergent, comme les activités de loisirs, les usages urbains ou ceux répondant à des fonctions environnementales.

Les activités de loisirs connaissent un fort développement lié à la présence du Grand site de SaintGuilhem-le-Désert et du Pont du Diable, classé au Patrimoine mondial par l'Unesco au titre des chemins de Saint-Jacques-de-Compostelle (800000 visiteurs/an), ce qui entraîne ponctuellement et/ou localement des situations de tensions. Les enjeux pour l'eau dans ce secteur concernent donc autant la satisfaction des besoins pour la consommation humaine que son utilisation à des fins de loisirs (base de canoë-kayak, baignade, etc.). La qualité des eaux de l'Hérault et donc du canal est étroitement surveillée dans le cadre de ces pratiques (DDASS) ainsi que les conditions de débits.

Les usages urbains représentent 8000 parcelles dont $25 \%$ en zone urbaine desservies par le canal de Gignac. Ces terrains urbains (maisons individuelles, lotissements) n'ont plus d'affectation agricole mais, soumis à une redevance foncière spécifique, appartiennent toujours au périmètre et doivent être desservis en eau brute à partir du réseau gravitaire existant. L'eau brute donne lieu parfois à la création d'un deuxième réseau d'alimentation géré par l'ASA, en parallèle à celui d'Adduction d'Eau Potable. La distribution d'eau brute est un très fort enjeu dans un contexte où un manque d'eau potable est prévisible à l'horizon cinq ans dans cette zone, comme l'indique le schéma directeur départemental d'AEP (Montginoul, 2006). À cette demande intra muros s'ajoute celle extra muros qui s'exprime à l'échelle du département et à travers un site emblématique : la source des Cent fonts. Les tests de pompages pilotés, durant l'été 2005, par le conseil général de l'Hérault, ont suscité une forte contestation locale. Le Sphaeromide raymondi, espèce de crustacé cavernicole rare et protégée à l'échelle européenne et dont l'habitat aurait, selon les opposants, été détruit par ces tests, est devenu le symbole de l'opposition pour les tenants d'une alternative à la mobilisation des ressources locales pour les besoins de la métropole régionale (association Prevhe, association pour la protection des ressources en eau de la vallée de l'Hérault).

Les usages « environnementaux » concernent à la fois les soutiens d'étiage, la réhabilitation des gravières et le maintien des zones humides. Concernant les premiers, il est, en effet, parfois nécessaire de procéder à des lâchers d'eau. "Cela se fait sans contrepartie car on ne sait pas aujourd'hui comment faire payer l'environnement » (entretien avec le directeur de l'ASA, 1997).

D'une réalisation technique grandiose, le canal est devenu, au fil du temps (sans que cet aspect ne s'efface), un outil agricole utile en même temps qu'un élément de création d'un territoire, par l'apparition de nouvelles fonctions ou l'élargissement des fonctions traditionnelles assumées par ce patrimoine hydraulique. Le canal de Gignac et l'organisme chargé de sa gestion, l'ASA, doivent faire face à plusieurs défis, parfois contradictoires. En ce qui concerne les prélèvements agricoles en eau, une certaine stabilité est à prévoir, conclut le SAGE. En matière de qualité, le dispositif des traitements des rejets reste à améliorer. Ainsi, à l'échelle du bassin de l'Hérault, $60 \%$ des 220 caves particulières n'ont pas de dispositif de traitement de leurs effluents. Pour les 40 caves coopératives, la question a été réglée grâce à des aides de l'Agence de l'eau et du Conseil général de l'Hérault. D'une façon générale, la situation observée est celle d'une baisse des pressions de pollution d'origine agricole du fait de la déprise et d'une amélioration des rejets.

L'utilisation modérée des possibilités d'irriguer est à rattacher à la fois à l'évolution générale des terres cultivées, aux habitudes locales et à des consignes nationales. Parmi les incertitudes, il sera intéressant de constater les effets du décret n²006-1527 du 4 décembre 2006 relatif à « l'irrigation des vignobles aptes à la production de vins à appellation d'origine » et qui permet aux exploitants de vignes AOC d'arroser ce qui était jusqu'alors interdit (ordonnance de 2000). Or, la zone dispose depuis 1985 d'une AOC « Côteaux du Languedoc ». Le nombre de demandeurs d'eau en période de tension sur la ressource est donc potentiellement en mesure d'augmenter. Un suivi sera utile lors des prochaines campagnes. En ce qui concerne les autres usages, en particulier les usages urbains, les demandes risquent d'être exponentielles. 
L'ASA doit en priorité réfléchir à la réforme en profondeur des systèmes d'irrigation : comment gérer les conflits entre utilisation individuelle et gestion collective? Dans quelle proportion faut-il substituer les réseaux sous pression et basse pression au système gravitaire ? À terme, cela pourrait permettre de mieux contrôler les débits prélevés ${ }^{22}$, donc d'optimiser la gestion de la ressource dans ce domaine comme au niveau de la distribution. Pour être mené à terme, ce projet demande des investissements exceptionnellement élevés, que la Moyenne Vallée de l'Hérault ne peut assumer seule. Les différentes collectivités doivent donc prendre la responsabilité de cette entreprise d'où de nombreuses interrogations dans un contexte législatif contraignant. Dans le débat engagé, les aspects identitaires et patrimoniaux ont leur place, au côté des aspects économiques, politiques, sociaux...

\section{Pressions sur les ressources, crise hydraulique et conscience commune de l'eau : éléments d'analyse}

Dans ce contexte, il s'agit donc d'identifier les germes des évolutions futures des modes de gestion.

\section{DE LA DIMENSION SOCIALE DES CRISES}

Les accélérations et crises sont souvent les moteurs du changement. Les ruptures relèvent de l'interaction entre les facteurs climatiques, les conditions sociales, politiques et économiques. Les réponses apportées sont souvent d'ordre technique et ne portent ainsi que sur la partie naturelle du phénomène. Elles supposent ainsi que la crise est un élément extérieur aux relations sociétéenvironnement et peuvent dans certains cas participer d'une construction sociale du manque d'eau (Jacob, 2005). La notion de crise peut être abordée de diverses façons. Certains privilégient l'angle socio-politique et philosophique : «la crise est plutôt considérée comme un phénomène endogène, comme une production imputable aux acteurs, aux organisations (qui ne sont pas face à la crise mais dans la crise). » (Beck et al., 2006, p. 4). Les perspectives de la longue durée et des interactions des sociétés avec leurs milieux, c'est-à-dire des développements sociaux et des processus biophysiques ont été étudiées par les sciences sociales (Jolivet, 1992; Levèque et Van der Leew, 2003). La crise est alors définie comme une discordance entre une dynamique sociale et celle du milieu biophysique. La capacité d'un système à intégrer dans son fonctionnement une perturbation, sans pour autant changer de structure qualitative désignée par le terme de résilience renvoie à divers types de comportements et permet en géographie de réfléchir aux notions d'instabilité, de robustesse, de vulnérabilité et de capacité d'adaptation (Aschan-Leygonie, 1998).

Dans le domaine de l'eau, la multiplication des pressions entraîne une crise hydraulique que l'on peut définir, à la suite de P. George (2004), bien qu'il se limite à la notion de crise en général, comme une rupture de rythme ou renversement de tendance dans une évolution. Les causes des crises sont complexes mais tiennent toutes à l'incapacité de contrôle total, souligne-t-il, insistant sur le fait qu'il s'agit d'épisodes évolutifs. Y. Lacoste (2003) rappelle l'étymologie du mot, Krisis, qui indique la décision et évoque les symptômes graves provoqués par les contradictions d'une évolution. B. Jobert (in Gilbert, 1990), propose d'en limiter l'usage à des situations précises celles

22. L'article 6 de la loi sur l'eau et les milieux aquatiques du 30 décembre 2006 (LEMA) a considérablement renforcé les obligations liées aux ouvrages situés dans le lit des cours d'eau en matière de débit réservé y compris pour les ouvrages anciens. La principale obligation consiste dans le respect du débit réservé fixé antérieurement à $1 / 40^{e}$ du module interannuel (moyenne interannuelle des débits moyennés sur l'année). À partir du $1^{\text {er }}$ janvier 2014, dans l'état actuel des textes, le débit réservé serait renforcé et passerait au $1 / 10^{\text {e }}$ en moyenne sur l'année avec possibilité de modulation tout en restant au minimum à $1 / 20^{\mathrm{e}}$. Le débit naturel des cours d'eau en zone méditerranéenne ne permet souvent pas de respecter cette nouvelle contrainte, les usages anthropiques tels que celui du canal de Gignac pourraient donc disparaitre. Élus, gestionnaires et utilisateurs se mobilisent actuellement pour que la reconnaissance de l'atypicité des cours d'eau méditerranéens soit prise en compte dans la rédaction du décret et que soit ainsi fixé des débits réservés plus bas (entretien avec le directeur de l'ASA de Gignac, mai 2007). 
Stéphane Ghiotti, Anne RiviÈre-Honegger

« dans lesquelles un système social est menacé dans son identité même ». Trois facteurs concourent de façon générale à la crise. La menace est reconnue comme l'un des premiers facteurs de crise. L'état normal se confond avec un sentiment collectif de relative sécurité. L'agent porteur est ici la pénurie. L'incertitude qui relève de la difficulté même d'identifier la menace est facteur de perturbation. Il s'opère un glissement entre le connu et l'inconnu, le maîtrisable, l'incompréhensible auquel se mêle un échec des différents savoirs et savoir faire technique, scientifique, politique, etc. La complexité, comprise à la suite d'E. Morin (1990) comme hétérogénéité, diversité et désordre est le troisième facteur. Elle reflète une situation où il y a trop de problèmes sans réponse, et où ces problèmes deviennent des accélérateurs de crise en suscitant l'intervention d'un grand nombre d'acteurs et de structures.

La crise doit être analysée comme un changement d'état d'un même système. Après avoir été considérée essentiellement sous l'angle d'une crise environnementale, soit en termes de raréfaction et de contamination de l'eau, la crise hydraulique est de plus en plus posée en liaison avec la question du développement humain durable par les mouvements associatifs et partiellement par les organisations internationales. Dans ce contexte, la question de l'eau se voit posée non plus seulement en termes de distribution mais aussi de répartition ce que Jean Bruhnes avait déjà mis en lumière dès le début du $\mathrm{xx}^{\mathrm{e}}$ siècle (Bruhnes, 1902). Cela implique un accès plus juste aux ressources, ces dernières étant considérées comme un droit humain fondamental à garantir. Les études de cas menées conjointement dans différents pays méditerranéens (CRIQUE, séminaires, 2006, notes personnelles; A. Gana et M. El Amrani, 2006) nous montrent, ainsi, que la crise hydraulique correspond largement à une crise des dispositifs de la gestion de l'eau. Cette crise, liée notamment à la redéfinition des usages prioritaires de l'eau et à l'accroissement des conflits entre anciens et nouveaux acteurs de l'eau redéfinit en même temps les bases sociales et territoriales de la gestion hydraulique ainsi que ses objectifs fondamentaux ${ }^{23}$.

\section{VERS LA CONSTRUCTION D'UNE CONSCIENCE COMMUNE?}

L'analyse du fonctionnement des ASA, à partir du cas du canal de Gignac, permet de dégager les éléments qui sont à la base de la construction de la conscience commune de l'eau et des crises des modes de gestion et de représentation des ressources qui sont liés à ses divers usages, dans la zone considérée. Elle fournit des éclairages intéressants sur leurs caractéristiques et les mécanismes de différenciation qui se développent entre différents groupes d'acteurs dans le temps. Crises et conscience commune sont en effet indissociables, leurs rapports mutuels n'étant compréhensibles que sur le temps long, tant les premières sont perçues et gérées à l'aune des expériences et des modalités de réponses au changement qui fondent la seconde (Beck et al., 2006). La conscience définie comme la connaissance des choses extérieures, par exemple un risque, résulte comme toute perception d'un processus complexe reposant à la fois sur des informations, des évènements, des jugements, des opinions, etc. Elle se construit de manière progressive au cours d'un processus combinant le triptyque «perception-représentation-action». Elle fait également référence à des interactions, des confrontations entre des consciences individuelles, des normes et des règles d'accès et de partage des ressources et un environnement donné dans une temporalité donnée. La mise en évidence et l'analyse d'une telle conscience implique de répondre à deux questions : conscience commune de quoi et conscience commune pour qui ? Il s'agit là de dégager des groupes d'acteurs susceptibles d'éprouver une conscience commune de l'eau et/ou des risques que peut engendrer son utilisation et le rôle de certains acteurs notamment dans la prise de conscience des risques

23. Nous menons actuellement une enquête par questionnaire auprès de 50 exploitants du périmètre de l'ASA de Gignac afin de mieux définir la crise hydraulique et d'identifier les risques environnementaux. Ce travail est conduit dans le cadre d'une recherche internationale dans un objectif de comparaison et associe six pays méditerranéens. "Risques et inégalités face à la gestion durable de la ressource hydraulique », Maison des Sciences de l'Homme, Paris, (2006-2009) Programme d'appui à la coopération pour la recherche en sciences humaines et sociales entre le Maghreb et la France coordonné par le Ladyss-CNRS. 
(rôle de catalyseur, d'intermédiaire, etc.). Cette conscience joue un rôle important en tant qu'élément de support de gestion en particulier dans le cadre de la gestion participative s'appuyant sur la concertation. La conscience commune connaît une évolution dans le temps, comme le montre l'exemple de l'ASA de Gignac où entretiens et recherche documentaire à partir des archives de l'ASA nous ont permis de distinguer trois phases liées en grande partie aux fonctions assurées par les réseaux hydrauliques (fig. 3). Tout d'abord, celle qui correspond à la situation d'origine, celle de la perception d'un risque commun par les agriculteurs (atteinte de la vigne par le phylloxera) et de la mise en place d'une structure de gestion collective à caractère d'établissement public en vue d'une mutualisation des coûts. Puis, s'inscrit la phase de la prise de conscience de l'évolution des besoins et de la demande en eau (eau ressource) provenant de systèmes hydrauliques mis en place. Cela se manifeste par la recherche de modalités d'intégration de nouveaux acteurs dans la prise en charge du fonctionnement de l'ASA. «Les urbains » représentent sur l'ensemble du périmètre $10 \%$ des surfaces, $50 \%$ des adhérents et leur part dans le budget est passé de $9 \%$ à $25 \%$ en quelques années, ce qui permet notamment le maintien du prix de l'eau agricole et par là même de la structure de gestion. Enfin, la phase que nous observons, celle de l'élargissement des fonctions et des acteurs, se traduit par le passage d'une approche centrée sur l'eau ressource à la prise de conscience de l'intérêt d'une vision globale, intégrée de l'eau-ressource/eau-milieu/eauterritoire ${ }^{24}$.

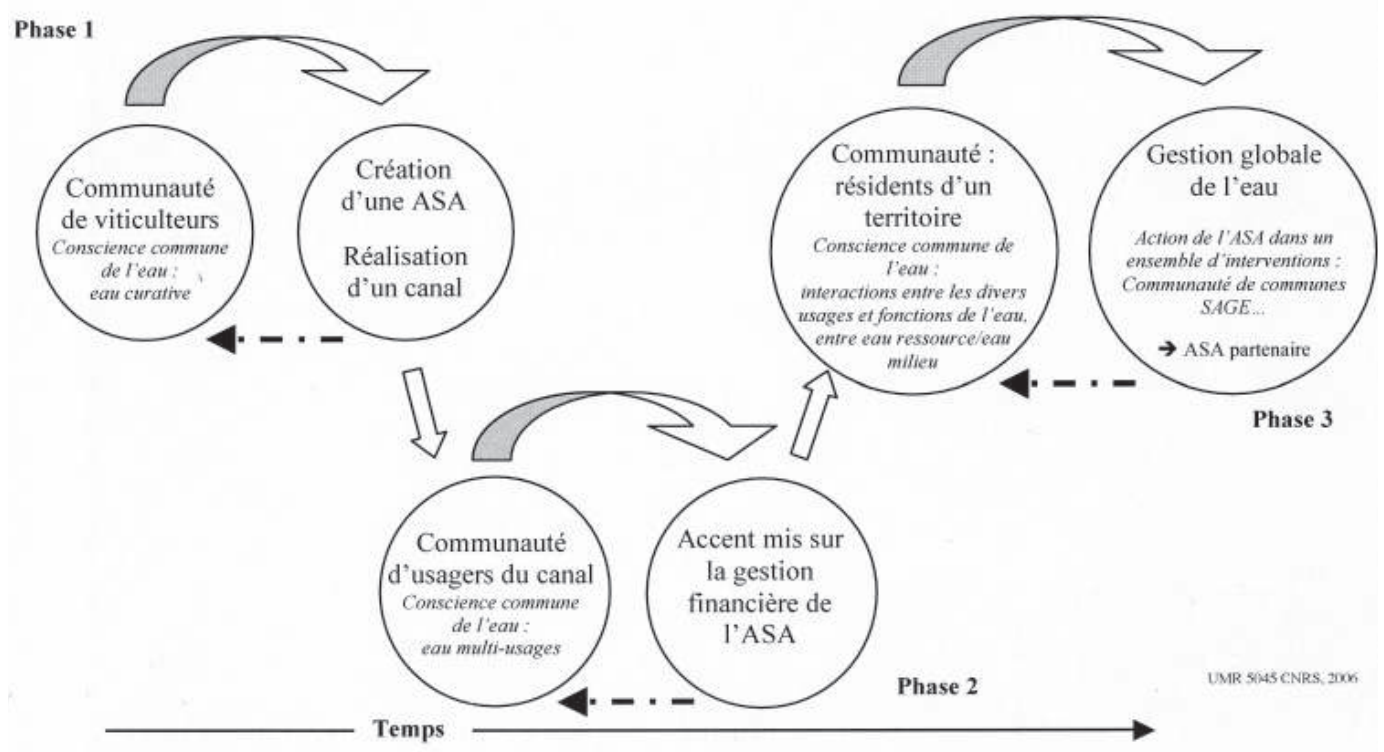

Figure 3 : Évolution de la conscience commune de l'eau Evolution of the common consciousness of the water

L'analyse de la mutation de la gestion des ASA (ou de l'évolution de leur mode de fonctionnement) est porteuse d'enseignements pour la mise en place d'une gestion intégrée de l'eau. Elle pose le problème de la pérennité d'une gestion concertée dans un contexte d'élargissement des fonctions assumées par les équipements hydrauliques concernés et permet d'en dégager les conséquences sur les modalités de financement et de leur prise en charge.

24. La distinction entre eau-ressource et eau-milieu repose sur un critère simple : lorsqu'elle fait l'objet d'un prélèvement, il s'agit d'eau-ressource; lorsqu'elle est utilisée in situ, on parle d'eau-milieu. 
L'évolution des conditions dans lesquelles s'exerce une telle gestion, en particulier le développement d'une conscience commune de l'eau et ses répercussions sur les modalités d'intervention, constitue une situation exemplaire pour dégager les éléments nécessaires ou favorables au développement d'une gestion concertée multifonctions. Si le risque semble être géré au niveau individuel (choix des cultures, mode d'irrigation, circuits commerciaux et de valorisation des produits, etc.), la crise relève de l'institution de gestion, l'ASA, puisqu'elle redéfinit les conditions d'accès et de partage de ressources. Elle est légitime dans cette mission puisque représentative d'un collectif partageant des intérêts communs (au moins sur la pérennisation du contrôle de la ressource). L'ASA tente ainsi de réajuster à la fois ses dispositifs techniques (micro-irrigation), ses instruments de gestion économiques (tarification) ainsi que ses modes de gouvernance. La mobilisation de ces différentes solutions « de sortie de crise », même si ces dernières sont plus ou moins imposées par des dispositifs réglementaires ou économiques, s’appuie sur une conscience commune de l'eau suffisamment partagée entre les membres du collectif ASA pour pouvoir être acceptée socialement et réellement mise en œuvre.

\section{Conclusion}

Dans la dynamique des interactions entre sociétés et environnement, les crises qui surviennent et redéfinissent les conditions d'accès, de partage et de gestion des ressources, ne sont pas des éléments extérieurs aux sociétés. L'adaptation, qui renvoie, à une conception plutôt déterministe de ces interactions n'est donc pas la seule solution possible. D’une manière générale, elle participe d'un ensemble de dispositifs mobilisables au même titre que l'innovation, l'appropriation ou le contournement comme l'indiquent de nombreux travaux sur l'irrigation méditerranéenne (ISIMM, 2008). Ces autres solutions mobilisent d'ailleurs beaucoup plus une certaine conscience commune de l'eau des sociétés locales qui s'appuie sur la richesse et la profondeur historique de leurs savoirs faire, là où l'adaptation mobilise souvent des solutions techniques de manière exclusive. Pour notre équipe, ces réflexions s'insèrent dans un travail plus large dont l'objectif est d'observer et d'analyser l'apport d'un système hydraulique ancien dans les préoccupations actuelles de maîtrise de l'espace à l'échelle d'un bassin-versant.

A l'échelle régionale, la réflexion sur « quelles stratégies pour quelles eaux pour le LanguedocRoussillon à l'horizon 2015? » doit donc s'appuyer sur trois questions indissociables les unes des autres :

- Quelles stratégies pour atteindre le «bon état écologique»? Cela passera nécessairement par la négociation des seuils, des normes, des débits etc.; ce qui, de façon pragmatique, peut être formulé par la question "Qui peut se payer le «bon état? "

- La territorialisation des politiques publiques et la décentralisation constituent-elles un remède à la fragmentation des territoires ou une accentuation de ce phénomène? ou «Qui va payer, combien et pour quelle eau? »

- Après la territorialisation, comment aborder la question de la différenciation territoriale? soit «A quelle échelle?»

En termes de perspectives, cela renvoie aux questions des liens entre d'une part, attractivité territoriale et transfert de durabilité et entre d'autre part, péréquation et solidarité territoriale, lesquels restent à approfondir. 


\section{Bibliographie}

Aschan-Leygonie (C.), 1998. La résilience d'un système spatial : l'exemple du Comtat. Une étude comparative de deux périodes de crises au XIXe et au XXe siècles, Thèse de doctorat, université Paris Panthéon-Sorbonne, $405 \mathrm{p}$.

Bas Rhône Languedoc, Région Languedoc-Roussillon, Conseils Généraux, 2006. Aqua 2020, Volet Ressources. Satisfaire les besoins en eau du Languedoc-Roussillon tout en respectant les milieux aquatiques, n.p.

Bas Rhône Languedoc, 2008. Un réseau hydraulique au service de l'aménagement du territoire un enjeu majeur pour le Languedoc-Roussillon, 16 p. [Consulté en ligne le 15 avril 2009 : http://www.brl.fr/index. php?id=255\&page $=6 \&$ rubrique $=20]$.

Beck (C.), Luginbuhl (Y.), Muxart (T.), 2006. Temps et espaces des crises de l'environnement, éd. Quae, $412 \mathrm{p}$.

Brunnes (J.), 1902. L'irrigation, ses conditions géographiques, ses modes et son organisation dans la Péninsule ibérique et dans l'Afrique du Nord, Paris, éditions C. Naud, 518 p.

CARRIÈre (I.), 1999. Évaluation économique du programme d'aménagement hydro-agricole du Bas-Rhône-Languedoc, Thèse de doctorat en économie, Université Montpellier 1, 363 p.

Conseil général de l'Hérault, 2005. Schéma d'aménagement et de gestion des eaux du bassin-versant de l'Hérault, Diagnostic et premières orientations. Présenté au Comité de bassin le 20 janvier 2006, 70 p. + 14 planches cartographiques.

—, 2005. Schéma d'aménagement et de gestion des eaux du bassin-versant de l'Hérault, Crues et inondations. État des lieux, cahier n² 2, 39 p. + 11 planches cartographiques.

—, 2005. Schéma d'aménagement et de gestion des eaux du bassin-versant de l'Hérault, Gestion quantitative de la ressource. État des lieux, cahier n 3, 37 p. +15 planches cartographiques.

—, 2005. Schéma d'aménagement et de gestion des eaux du bassin-versant de l'Hérault, Gestion qualitative de l'eau et des milieux aquatiques. État des lieux, cahier nº 4, 48 p. + 18 planches cartographiques.

DiREN Languedoc-Roussillon, 1999. L'environnement en Languedoc-Roussillon, 262 p.

Ferras (R.), 1998. 99 réponses sur... Le Languedoc-Roussillon, CRDP, Montpellier.

Gana (A.), El Amrani (M.), 2006. « Crise hydraulique au Maghreb : raréfaction de la ressource ou problèmes de gestion? ", Géocarrefour, vol. 81, n 1, p. 37-50.

Garin (P.), Loubier (S.), 2002. Durabilité et fragilité des Associations Syndicales Autorisées (ASA) d'irrigation en France, compte rendu pour l'Académie de l'Agriculture, Fr nº 88 (3), p. 61-71.

GENIN (B.), 2005. Quelle politique de l'eau pour quel aménagement du territoire en Languedoc-Roussillon, note de synthèse, DIREN Languedoc-Roussillon, 8 p.

George (P.), Verger (F.), 2004. Dictionnaire de la Géographie, PUF, coll. «Quadrige », 474 p.

Gнiотті (S.), 2007. Les territoires de l'eau. Gestion et développement en France. Paris, CNRS Éditions, $246 \mathrm{p}$.

INRA, 2006. Sécheresse et agriculture : réduire la vulnérabilité de l'agriculture à un risque accru de manque d'eau, rapport d'expertise collective, n.p..

ISIIMM (inNOVATIONS SOCIALES ET INSTITUTIONNELLES DANS LA GESTION DE L'iRRigaTiON EN MÉditerRANÉE), 2008. Synthèse générale, 113 p.

Jarrige (F.), Thinon (P.), Nougaredes (B.), 2006. « La prise en compte de l'agriculture dans les nouveaux projets de territoires urbains. Exemple d'une recherche en partenariat avec la communauté d'agglomération de Montpellier », Revue d'Economie Régionale et Urbaine, n 3, p. 393- 414.

Jacob-Rousseau (N.), 2005. «Aspects de la pénurie hydrique et de sa gestion dans la Cévenne vivaraise au XIX siècle ", Géocarrefour, vol. 80, n 4, p. 297-308.

Jobert (B.), 1990. «Introduction », dans Gilbert (C.) (dir.), La catastrophe, l'élu et le préfet, PUG, Grenoble.

Jollivet (M.) (éd.), 1992. Sciences de la nature, Sciences de la société. Les passeurs de frontière, Paris, CNRS Éditions, 589 p.

Lacoste (Y.), 2003. De la géopolitique aux paysages, Dictionnaire de la géographie, Paris, Armand Colin, $413 \mathrm{p}$. 
Levêque (C.), VAn der Leeuw (S.), (dir.), 2003. Quelles natures voulons-nous? Pour une approche socio-écologique du champ de l'environnement, Paris, Elsevier.

Montginoul (M.), 2006. «Les eaux alternatives à l'eau du réseau d'eau potable pour les ménages : un état des lieux », Ingénieries, n 45, Cemagref, p. 49-59.

Morin (E.), 1990 (rééd. 2005). Introduction à la pensée complexe, Point, coll. « Essais », 158 p.

Parde (M.), 1925 (rééd. 2004). Le régime du Rhône, thèse de doctorat, Revue Géocarrefour, Lyon, 848 p.

Pôle prospective, 2007. Futurs possibles, impacts sur les territoires, cahier 2 - Devenir de l'agriculture, Prospectives des territoires en Languedoc-Roussillon - Maintenir l'attractivité des grands territoires urbains, CNRS, Montpellier, $72 \mathrm{p}$.

Puech (D.), 2001. Évaluation économique de la valeur patrimoniale des réseaux d'irrigation gravitaire », Journées techniques agriculture/environnement, «Irrigation gravitaire », Avignon, p. 212-219.

Puech (D.), Rivière-Honegger (A.), 2001. - État des lieux des Associations Syndicales Autorisées en Languedoc-Roussillon, DIREN Languedoc-Roussillon, 5 tomes, dont rapport, 120 p. (inventaire mis à jour en 2003)

Puech (D.), Rivière-Honegger (A.), Ghiotti (S.), 2006. Le rôle des associations de propriétaires fonciers (ASA) dans la dynamique de la perception et de la gestion des risques environnementaux. L'exemple de l'ASA du canal de Gignac (Hérault-France), rapport final, programme "Eau, environnement, société », CNRS, « Crise hydraulique et perceptions du risque environnemental en Méditerranée occidentale », 81 p. + annexes.

Rivière-Honegger (A.), 2001. Surfaces-ressources en Languedoc-Roussillon, Journées techniques agriculture/environnement, « Irrigation gravitaire », Avignon, p. 22-32.

—, 2004. La gestion de l'eau par les associations de propriétaires fonciers. Méthodologie pour un inventaire régional, dans Ruf (Th.), Rivière-Honegger (A.), La gestion sociale de l'eau, concepts, méthodes et applications, Territoires en mutation, $\mathrm{n}^{\circ} 12$, p. 63-80.

Cet article a été reçu le le 5 juillet 2008 et a été définitivement accepté le 28 mai 2009. 